\title{
Feuille, baraque, écorce, bordel. Claude Simon à Mühlberg sur Elbe
}

\section{Marcel Beyer}

\section{(2) OpenEdition}

\section{$\checkmark$ Journals}

Édition électronique

URL : http://journals.openedition.org/ccs/973

DOI : $10.4000 /$ ccs. 973

ISSN : 2558-782X

Éditeur :

Presses universitaires de Rennes, Association des lecteurs de Claude Simon

\section{Édition imprimée}

Date de publication : 30 avril 2015

Pagination : 223-234

ISBN : 9782753539990

ISSN : 1774-9425

Référence électronique

Marcel Beyer, "Feuille, baraque, écorce, bordel. Claude Simon à Mühlberg sur Elbe », Cahiers Claude Simon [En ligne], 10 | 2015, mis en ligne le 22 septembre 2017, consulté le 15 septembre 2020. URL http://journals.openedition.org/ccs/973 


\title{
FEUILLE, BARAQUE, ÉCORCE, BORDEL. CLAUDE SIMON À MÜHLBERG SUR ELBE
}

\author{
Marcel BEYER
}

Dans la scène originaire des récits de Claude Simon, où celui-ci revient inlassablement sur une journée de mai 1940, un personnage appelé tantôt Georges, tantôt "il » ou "je ", chevauche derrière son colonel, entre deux hameaux des Ardennes, adoptant sans s'en rendre compte le rôle de Sancho Panza. Le matin du 17 mai 1940, comme le précise Simon dans L'Acacia, ce Sancho Panza voit son Don Quichotte, qui porte ici le nom de Reixach, tomber de l'espace romanesque dans la réalité meurtrière et se faire tuer dans une embuscade. Les temps ayant changé, Don Quichotte n'est plus déguisé en chevalier, mais en capitaine de la cavalerie française; néanmoins, plus de trois cents ans après, il est toujours assis sur un cheval brandissant un sabre de manière grotesque au moment même de sa mort.

Dans L'Acacia, l'auteur le qualifie d'" anachronisme équestre en train de brandir son sabre ", et on a l'impression que, depuis la première apparition de Reixach en 1947 dans La Corde raide, Simon a transformé délibérément, au cours d'un processus de plusieurs décennies, l'apparence physique du capitaine de cavalerie pour en faire un chevalier à la triste figure. Dans ses Excursions à travers les paysages romanesques de Claude Simon, Ralf Vollmann a retracé en détail cette transformation progressive. Au début, c'était un aristocrate hautain, fidèle à ses principes, sans doute un peu étranger au monde; à la fin, c'est un personnage qui " chevauchait maintenant à cru un lourd et malpropre sous-verge d'un attelage de mitrailleuse [...] dont les traits 
sectionnés (ou décrochés - ou rompus) pendaient à terre avec un léger tintement (celui d'une boucle défaite?) de chaque côté de la croupe où ils laissaient dans la poussière un double sillage ", un officier qui "était selon toute apparence devenu fou ", comme il est dit dans L'Acacia.

Le Sancho Panza du matin du 17 mai 1940, qui suit avec une obéissance aveugle son supérieur dans le monde réel, n'aura plus l'occasion d'expliquer à Don Quichotte la différence, en l'occurrence vitale, entre réalité et imagination, et, de ce fait, il manquera l'unique mission que son personnage doit accomplir conformément au modèle littéraire. Se rapportant à un officier de cavalerie qui est d'abord tombé de l'espace romanesque, puis, juste après, de son cheval, cette différence réside dans le fait que derrière les moulins à vent imaginaires se cachent de vrais géants, à savoir, comparés aux cavaliers français brandissant leur sabre, des Allemands surpuissants, des tireurs d'élite équipés de mitrailleuses, qui sont dissimulés derrière d'anodines haies agrémentant le paysage.

« Il », « Georges » ou " je », qui dans l'intervalle est en route depuis longtemps pour le camp de prisonniers, se retrouve seul face à l'obstacle existentiel que représente le rapport existant entre la réalité et les images que nous nous faisons d'elle. Et c'est précisément à cet endroit que, depuis son roman le plus connu paru en 1960, La Route des Flandres, il est convenu de situer la naissance du romancier Claude Simon.

Monté sur son âne, le Sancho Panza du début du XviI ${ }^{\mathrm{e}}$ siècle tournait le dos à l'époque des aventures médiévales, tandis que le Sancho Panza du milieu du $\mathrm{xx}^{\mathrm{e}}$ siècle plonge tout droit dans l'aventure de l'écriture.

J'ignore si les critiques littéraires et les universitaires lisent les romans de Claude Simon à la lumière des évènements contemporains de leur genèse, s'ils replacent par exemple Le Palace paru en 1962 dans le contexte de la guerre d'Algérie, La Bataille de Pharsale de 1969 dans celui de la guerre du Vietnam et Le Jardin des Plantes de 1997, que Claude Simon consacre à celui qui a été surnommé le " renard du désert ", Erwin Rommel, dans le contexte de cette autre guerre du désert connue sous le nom de deuxième guerre du Golfe ou de première guerre d'Irak.

Non pas que je sois d'avis que l'on puisse trouver dans les romans de Simon des traces concrètes de ces guerres qui ont dominé notre époque alors que, dans son écriture, il se tourne vers des guerres passées. Mais comme bruit de fond, comme évènements perçus quotidiennement au moins sous forme d'images, je 
me dis qu'il est tout à fait possible qu'elles aient contribué à déclencher l'écriture de la mémoire: le theater of war, comme on dit depuis Clausewitz, avec ses theaters of operation, que les soldats engagés dans le désert appellent familièrement sandboxes, fait surgir des images des évènements actuels au contact desquelles la mémoire s'enflamme en un processus sans fin, qui se poursuit de roman en roman.

En tout cas, il n'y a guère d'autre lecture possible de Claude Simon, cet auteur qui a décliné sous toutes ses formes la guerre au $\mathrm{xx}^{\mathrm{e}}$ siècle, si l'on ne veut pas réduire ses réflexions esthétiques à de simples observations relevant de l'histoire de l'art et de la littérature. Dans un commentaire portant sur une série de photos publiées dans le numéro de la revue suisse $D u$ qui lui est consacré, Simon écrit en 1999: "Le lieu n'est pas ici d'analyser en profondeur l'émergence d'un phénomène qui semble toutefois inséparable (mise en question d'un certain "humanisme", d'une certaine "logique", dislocation des structures sociales, etc.) des convulsions profondes qu'a traversées le monde - en particulier l'Europe - depuis le début du siècle et ont conduit aussi bien plasticiens qu'écrivains à rejeter un certain nombre de conventions établies en cherchant dans cette apparente "destruction" (de la morale, de la raison, du modèle, du "sujet", de l'histoire racontée) un nouvel ordre, une autre harmonie, une autre logique. "

Lire "les convulsions profondes qu'a traversées le monde - en particulier l'Europe - depuis le début du siècle " comme un bilan historique, comme l'analyse d'une époque achevée, ferait de Claude Simon un auteur de romans historiques, ce qu'il n'est certainement pas. Il ne récapitule pas. L'écriture se poursuit comme se poursuivent les convulsions, et tout comme les convulsions, l'écriture est soumise à des changements. Se souvenir est, à chaque époque, un processus qui s'accomplit au présent.

En automne 1992, alors que je découvrais Claude Simon pour la deuxième ou troisième fois, et donc pour toujours, je passai six mois à Berlin au bord du lac de Wannsee: dans la journée, je travaillais à mon roman Voix de la nuit (Flughunde), le soir, je partais pour Berlin-Mitte, puis la nuit, la tête pleine des images de ce que je venais de vivre dans l'immédiat, images qui provenaient elles-mêmes d'au moins deux époques entre-temps révolues, mais qui débordaient sur le présent et le futur, la nuit donc, je lisais L'Acacia.

Je lisais à la lueur de ma lampe de chevet, tandis qu'au-dessus de moi une inquiétante tache noire apparaissait au plafond - une araignée explorait la chambre 
où elle avait élu domicile pour ses quartiers d'hiver. Nous étions en septembre, le mois où, comme je l'appris dans Der Radardenker (Le Penseur radar) de Gottfried Benn, on trouve tous les matins " un de ces géants sur le mur ou dans un des lavabos ", et comme je poursuivais ma lecture de Claude Simon avec Les Géorgiques, je m'émerveillai de découvrir un de ces fils invisibles qui permettent de relier des livres provenant d'époques, de langues et de sphères différentes, car chez Simon aussi, il est question dès le début de l'étonnante invasion annuelle des araignées de septembre.

Mais revenons aux circonstances qui ont présidé à l'écriture du roman: on pourrait penser aujourd'hui qu'il était évident en 1989, au moment de la parution de L'Acacia, de lire le roman à la lumière de la fin imminente de la guerre froide. Après tout, l'auteur avait vécu personnellement un moment décisif de la perestroïka et en avait fait le thème principal de son roman précédent, L'Invitation. Il est même possible que Claude Simon, témoin, lors d'un voyage en Union soviétique, de la tentative du socialisme tardif de rallier des artistes internationalement reconnus aux réformes engagées par Gorbatchev, ait été le premier écrivain, à partir de son observation personnelle, à faire de Mikhaïl Sergueïevitch Gorbatchev un personnage littéraire.

Pour le lecteur germanophone du reste, ce portrait de Gorbatchev est lié à un moment de l'histoire interallemande: dans la traduction allemande de L'Invitation, parue en 1988 à Reinbek près de Hambourg, on apprend, certes, que Simon avait suivi avec un mélange d'ennui et d'irritation le discours tenu par Gorbatchev devant les participants au Forum d'Issyk-Koul. Si nous voulons toutefois nous faire nous-mêmes une idée de ce discours, il nous faut consulter le numéro 3/ 1987 de la revue Sinn und Form publiée à Berlin-Est.

Par la suite, de nouvelles convulsions devaient secouer le monde, et l'Europe en particulier, des structures sociales entières devaient disparaitre. Autant que je me souvienne, les critiques littéraires bien souvent avides d'actualité à en devenir rébarbatives ne mentionnaient la transformation de l'Europe ni en 1989, ni en 1991 lorsque parut la traduction allemande de L'Acacia. Claude Simon ne semblait prendre en considération "comme toujours" que les convulsions de la première moitié du siècle. Il n'y était nulle part suggéré que ce romancier, qui déclinait toutes les formes de la guerre, ait pu le faire cette fois-ci sous un nouveau jour.

L'image que j'avais de Claude Simon se transforma néanmoins à la lecture de L'Acacia. Jusque-là, c'était quelqu'un qui composait ses romans en faisant une confiance absolue aux images qui naissaient dans ma tête. Quelqu'un qui, comme nul autre, hissait l'art de la périphrase juste, tout comme celui de l'ellipse efficace 
parce que précisément discrète, à des sommets d'où je pouvais plonger dans des abîmes d'autant plus profonds de la connaissance. Quelqu'un dont le style, malgré sa flexibilité, s'opposait admirablement à toute rhétorique du dimanche, ou plus exactement: dans l'univers duquel la rhétorique du dimanche n'existait tout simplement pas. Et bien sûr il devait s'agir d'un écrivain remarquable, car en fin de compte il avait inspiré à Eva Moldenhauer dans sa traduction vers l'allemand des phrases vertigineuses qui restituaient l'obscurité en une clarté parfaite. Or L'Acacia était bien plus que cela: avec ce livre, on passait d'une lecture stimulante, réellement instructive, à un outil de perception du monde par le biais de l'écriture.

Je passais des heures sur la Potsdamer Platz, qu'on pouvait à peine qualifier de place à l'époque, je regardais les restes du Mur et de la "zone interdite " avec son large chemin de ronde et ses réverbères à la lumière trop vive, je regardais ce vaste terrain en partie bourbeux, en partie envahi par des buissons bas, animé uniquement par un petit cirque ambulant, terrain où se dressait autrefois la Chancellerie $\mathrm{du}$ Reich et sous lequel se trouvaient encore les restes du bunker dans lequel Adolf Hitler avait passé ses derniers jours.

Je me promenais entre les strates du temps et je voyais de plus en plus dans L'Acacia un outil grâce auquel je commençai, je recommençai une nouvelle fois "à rejeter un certain nombre de conventions établies en cherchant dans cette apparente "destruction" (de la morale, de la raison, du modèle, du "sujet", de l'histoire racontée) un nouvel ordre, une autre harmonie, une autre logique", pour reprendre la formulation de Claude Simon.

Et dans L'Acacia lui-même, je trouve aussi ce recommencement, dans un autre contexte historique, car il convenait de considérer l'Europe dans une nouvelle perspective, en particulier dans le douzième chapitre. La simple référence que Simon fait à Proust au début de cette dernière partie, ainsi qu’à son propre livre écrit après la guerre, $\mathrm{La}$ Corde raide, annonce un texte sur les conditions de l'écriture. Simon esquisse une nouvelle fois sa poétique, ou plus exactement: il fait du lecteur l'observateur d'un processus au cours duquel la poétique se déploie sans pour autant fournir des formules toutes faites.

IV

Le Don Quichotte du 17 mai 1940 a donc laissé son Sancho Panza affronter seul l'obstacle existentiel du rapport entre la réalité et les images que nous nous faisons d'elle, et « lui », comme il est appelé systématiquement dans L'Acacia, doit 
affronter cet obstacle perfide sans son interlocuteur enfermé dans des mondes imaginaires.

Je voudrais procéder ici à un petit glissement et détourner l'attention des moulins à vent qui n'existent de toute façon que dans les vieux romans, pour me tourner plutôt vers leurs fondations, par nature peu solides, qui n'indiquent en réalité rien d'autre qu'un vide et qui, en outre, contrairement à ce que suggère leur nom, ne représentent pas une éminence dans le paysage, mais s'intègrent presque imperceptiblement dans une plaine, en tant que lieu se situant, pour reprendre Claude Simon, dans « la plaine sablonneuse de Saxe »: Mühlberg ${ }^{1}$.

Il s'agit du camp de prisonniers de guerre de Mühlberg - plus précisément: du camp pour hommes de troupe, Mannschafts-Stammlager IV B, en abrégé Stalag IV B, Mühlberg sur Elbe - où Simon a été interné cinq mois en 1940. C'est ici, je pense, que l'auteur transpose dans L'Acacia la scène originaire du romancier Claude Simon. Qui plus est, il décompose petit à petit la scène initiale de la mort du capitaine de cavalerie, autour de laquelle tournent également plusieurs chapitres de ce roman, et il le fait par si petites touches, en décalant de si petits tableaux les uns par rapport aux autres, que la dissolution complète de la scène qui lui est devenue chère au cours de trois décennies risque de passer inaperçue, même aux yeux de ses lecteurs de longue date. Ce n'est plus une image, un évènement qui feront finalement de Claude Simon un écrivain, mais une suite d'évènements qui se déroulent sur une toile de fond changeante et à des époques historiques ayant connu différents retentissements.

$\mathrm{V}$

Dans le dernier chapitre nous voyons à trois reprises le personnage principal se pencher sur une feuille de papier afin de la recouvrir de lignes tracées au crayon. La troisième fois cependant, à la fin du roman, la feuille reste blanche parce que la scène, devenue célèbre, où l'auteur apparaît à l'instant qui précède l'acte d'écriture, est figée, arrêtée: "un soir il s'assit à sa table devant une feuille de papier blanc ». Ce sur quoi, sans suivre la chronologie de l'œuvre, mais en obéissant à la logique interne, il faudrait lire à nouveau la première page de L'Acacia.

À cette image fixe fait écho un portrait sarcastique de George Orwell qui se trouve dans le roman précédent de Simon, Les Géorgiques: "Se le figurer donc, assis devant une table (il ne dit pas où, sauf que c'était de nouveau l'Angleterre),

1. Dans ce passage où il est question de moulins à vent et de la plaine de Saxe, Marcel Beyer joue avec le nom "Mühlberg " qui signifie en allemand: « montagne au moulin » [NdT]. 
la table peut-être devant une fenêtre ouverte, peut-être sur la paisible campagne, ou une lande plantée de bruyères, ou la mer, ou une rue ".

La scène, tout d'abord imaginaire, qui semble anticiper l'image finale de L'Acacia, se révèle, quelques pages plus loin, être une façon acerbe de se démarquer du projet d'écriture d'Orwell: "En fait ", écrit Simon, - c'est-à-dire: "au fond", "en réalité", peut-être aussi "objectivement" ou "de facto"-, « il est constamment préoccupé de l'effet produit. Assis là à sa table, ce sera comme s'il parlait tout haut dans le silence, s'interrompant peut-être de temps à autre pour porter la main à son cou, toucher la cicatrice de la balle qui lui a à moitié sectionné les cordes vocales comme pour s'assurer qu'il n'a pas rêvé, puis se remettant à écrire; ou plutôt à se parler de sa voix blanche, voilée, semblable à un halètement, épiant son invisible auditoire de morts et de vivants à la fois incrédule et attentif, composé de personnages qui sont à quelques variantes près comme autant de copies conformes de lui-même."

Dans le passage allant de "Se le figurer " à "En fait, il est constamment préoccupé de l'effet produit", Simon ne pourrait pas, compte tenu de sa propre poétique, faire un portrait plus ambivalent d'un collègue écrivain. Lui qui ne nous montre, à nous lecteurs, que des images, et qui n'éprouve presque jamais le besoin de regarder dans la tête de ses personnages, et qui, s'il le fait, ne nous livre que des images-souvenirs ou des perceptions, sans jamais toutefois nous trahir leurs intentions secrètes, bat ici l'adversaire avec ses propres armes: il met en scène une « connaissance " que le narrateur aurait de la " vérité » qui constitue pour lui une source de détestation, ce qui n'échappe pas à ses lecteurs. Le fait qu'à la fin de L'Acacia, le personnage principal s'installe à sa table, signifie un abandon radical de toute intention préalable à l'utilisation d'une feuille blanche.

VI

Mais cet abandon de toute intention préalable est lui-même le résultat d'un processus qui a été déclenché dans le camp de prisonniers de Mühlberg. Simon entreprend, du moins pour la première fois dans L'Acacia, de le situer dans la plaine sablonneuse de Saxe.

"Pendant des mois, chaque dimanche, il avait exécuté au crayon des dessins que par l'intermédiaire d'un souteneur juif d'Oran il vendait à leurs gardiens contre les paquets de tabac qui constituaient la monnaie du camp ", est-il dit à la première image où le personnage principal se penche au-dessus d'une feuille de papier, dans la seule intention de faire du dessin pornographique en cours un 
dessin aussi fidèle et suggestif que possible, "se demandant parfois - puis cessant même de se le demander - comment on pouvait bien payer des sommes aussi énormes que trois ou quatre paquets de tabac (ce qui, dans l'univers où il vivait, représentait une petite fortune) pour des choses aussi dénuées d'intérêt tant à ses yeux qu'à ceux du souteneur oranais ".

Il est révélateur qu'il se souvienne de cet épisode après son évasion du camp de prisonniers, alors qu'il se trouve dans un bordel à côté d'une prostituée; s'interrogeant sur la manière dont l'une des situations se reflète dans l'autre, il se voit " incapable de formuler ce qu'il éprouvait ", car le " souteneur oranais » était aussi dans une certaine mesure le sien, il était la mère maquerelle du petit magasin de fournitures pour le dessin qu'il tenait avec l'aide de son protégé talentueux, l'un étant responsable de la satisfaction du désir sexuel, tandis que l'autre, comme l'écrit Simon, "allait proposer à leurs gardiens les corps nus et fornicateurs patiemment dessinés chaque dimanche sur les feuilles de papier qu'il (l'Oranais) se procurait on ne savait comment dans un quadrilatère entouré de triples barbelés ".

Soit dit en passant, il est assez curieux d'imaginer que dans la succession d'un ancien soldat de la Wehrmacht, peut-être même originaire de Saxe, puissent se trouver les premières œuvres graphiques du Prix Nobel de littérature de 1985, œuvres qui seraient contemplées aujourd'hui avec étonnement par ses petits-enfants ou arrière-petits-enfants.

Dans l'alternance, qui court sur plusieurs pages, des images du bordel avec les souvenirs d'un compagnon de captivité qui écoule ses dessins, on trouve inséré un court passage dans lequel Simon décrit le personnage principal, " la lumière éteinte, écoutant la respiration régulière autour de lui, toujours gisant, [...] pensant de nouveau: "Bon Dieu, bon Dieu, bon Dieu!...", pensant aux corps empilés dans l'épaisse puanteur de la baraque, au grouillement des poux, aux barbelés, aux silhouettes échassières des miradors avec leurs montants décharnés de pin brut, vineux, sommairement dégrossi, comme écorchés, hérissés de place en place de languettes d'écorce"

Il s'agit là d'une image provenant du camp de prisonniers, cela ne fait aucun doute, et pourtant elle évoque d'autres images qui montrent d'autres camps, d'autres " corps empilés ». Fin 1940, il aurait été impossible de se souvenir de ces images, que ce soit dans la réalité ou dans un roman de Claude Simon, parce que celui-ci n’a pas écrit de romans dont les personnages connaissent les évènements qui vont se produire dans un proche avenir. Il se peut par contre que l'auteur les ait eues à l'esprit en écrivant, ainsi que le lecteur qui, comme moi, se plonge régulièrement dans L’Acacia depuis le début des années 90. 
Un jour, écrit Simon, "l'Oranais fut envoyé en commando (pas en punition, pas en tant que juif non plus quoi qu'il ne s'en cachait pas, s'en enorgueillît même, lui qui de sa vie n'avait probablement jamais mis les pieds dans une synagogue, jamais observé Kippour) », et le personnage principal le perd de vue sans qu'il ne revienne ni ne soit mentionné une seule fois.

Feuille, baraque, écorce, bordel: les scènes se passent, comme je l'ai dit, dans la deuxième moitié de l'année 1940, à l'époque où il était peut-être encore possible à un prisonnier de guerre français détenu en Saxe de révéler son identité juive, ne serait-ce qu’à ses codétenus.

VII

Bientôt "il ", le personnage principal, se met à dessiner non plus les habituelles scènes pornographiques mais des feldwebel recouverts de médailles, puis il parvient à s'évader. Il fréquente alors le bordel où il a sous les yeux les "corps empilés dans l'épaisse puanteur de la baraque ». "Un jour il acheta cependant un carton à dessin, du papier, deux pinces et, au cours de ses promenades, il s'asseyait quelque part et entreprenait de dessiner, copier avec le plus d'exactitude possible, les feuilles d'un rameau, un roseau, une touffe d'herbe, des cailloux..." ", comme s'il voulait recouvrir les images-souvenirs à l'aide de ses yeux et de sa main, comme s'il voulait mettre quelque chose à leur place.

Peu de temps après, nous le voyons un soir, assis à sa table devant une feuille de papier blanc, dans l'attente des images qui sont susceptibles de venir, qu'il s'agisse du feuillage de l'acacia devant la fenêtre ou précisément de ces corps empilés dans la baraque.

C’est cela, pour Claude Simon, être écrivain.

Écrire signifie aussi ne pas s'opposer à la superposition des images, même si elles proviennent de divers contextes d'expérience, de différentes strates temporelles ou de sphères médiatiques spécifiques: l'image ultérieure empiète sur la précédente et la transforme, l'image télévisée d'une actuelle convulsion violente qui secoue le monde a des répercussions sur la mémoire corporelle que nous croyons incorruptible.

Après la fin de la guerre froide et après L'Acacia où il décrit pour la première fois le "souteneur juif d'Oran " qui, semaine après semaine, le fournit en feuilles de papier blanc, Claude Simon fera du peintre Gastone Novelli, qui a été torturé à Dachau, la figure centrale de son roman Le Jardin des Plantes. Et dans le commentaire accompagnant la série de photographies parues dans la revue $D u$, il 
révèlera, avec ce sens de la remarque incidente qui lui est propre, que le portrait auquel ses lecteurs associent jusqu'à aujourd'hui le visage du jeune Claude Simon est précisément celui qu'il a fait réaliser au printemps 1937, comme il le décrit dans L'Acacia, chez un photographe resté anonyme du ghetto de Varsovie, parce qu'il avait besoin de photographies d'identité sans lesquelles il n'aurait pas obtenu de visa pour l'Union soviétique.

Claude Simon ne pourrait s'éloigner davantage de l'« anachronisme équestre en train de brandir son sabre ", anachronisme qui en restera toujours un parce que, dans son univers, il n'existe pas de camps d'extermination dont les images puissent se superposer aux images d'un camp de prisonniers de guerre.

\section{VIII}

Je me suis rendu moi-même plusieurs fois à Mühlberg sur Elbe à la fin des années 90 pour voir l'emplacement de l'ancien Stalag IV B. On roule environ une demi-heure depuis Dresde en direction de Riesa, puis avant le fleuve, on tourne à droite en passant sous la ligne de chemin de fer et on suit encore un moment la route qui s'étend en effet dans un paysage uniformément plat, ouvert dans toutes les directions. Il y avait à l'époque sur le bas-côté - j'ai failli dire: à intervalles réguliers - un nombre effrayant de croix de bois ornées de fleurs et pourvues de plaques portant les noms de jeunes gens qui s'étaient probablement tués en voiture à cet endroit.

C'est donc dans ce monotone paysage sablonneux de Saxe, pensai-je, que Claude Simon, pendant ses cinq mois de captivité, a creusé un puisard, entreprise peu aisée, ces camps n'étant pas construits sans raison sur un sol sablonneux où toute tentative de creuser un tunnel pour s'évader est vouée à l'échec.

Quand je vois aujourd'hui des travaux d'excavation quelque part aux alentours de Dresde, je pense à Claude Simon en train de creuser un puisard, au souteneur juif d'Oran lié à son personnage principal et aux histoires que l'on peut entendre lors des fêtes villageoises de la région, à condition que l'accent ne révèle pas clairement que l'on est originaire d'Allemagne de l'Ouest et que l'on est venu s'installer après 1989 . S'ils ont suffisamment bu, les agriculteurs racontent ce que leur racontaient sur la fin de la Seconde Guerre mondiale les valets de ferme des anciens grands fermiers lorsqu'ils avaient eux-mêmes suffisamment bu.

Au printemps 1945, alors que, de l'Ouest approchaient les troupes américaines et de l'Est l'Armée rouge, sans que l'on ait pu savoir laquelle des deux armées arriverait la première, certains grands fermiers qui pendant la guerre avaient 
plutôt bien vécu, mais avaient d'autant plus maltraité « leurs » travailleurs forcés, prirent peur. C'est pourquoi, avant qu'ils ne puissent raconter leur histoire, donner des noms et désigner ceux qui les avaient maltraités, on fit sortir des fermes les travailleurs forcés, en particulier ceux qui étaient originaires d'Europe de l'Est, car c'est la vengeance de l'Armée rouge que l'on craignait le plus, on les tua à l'abri des regards dans un bosquet ou au creux d'une dépression de terrain invisible depuis la ferme, et on enterra leurs cadavres dans le sol redevenu meuble après les derniers gels.

Traduit de l'allemand par Laurent Cassagnau 
Marcel Beyer, né en 1965, a suivi des études de lettres à l'université de Siegen. Après ses études, il a travaillé comme lecteur du magazine littéraire Konzepte (1990-1993) et il a contribué au magazine de musique Spex (1992-1998). En 1996 et en 1998, Beyer a été élu "Writer in residence " au University College de Londres et à l'université de Warwick à Coventry. Depuis 1996, Beyer vit à Dresde. Influencé par des écrivains comme l'autrichienne Friederike Mayröcker (sujet de sa thèse de maîtrise) ou par le Nouveau Roman, Marcel Beyer écrit de la poésie, des essais et des romans. Il est lauréat de nombreux prix littéraires prestigieux et l'un des romanciers les plus connus de sa génération. Avec son deuxième roman Flughunde (1995) commence une série d'ouvrages dédiés à l'histoire de l'Allemagne, dans lesquels Beyer mélange fiction et documentation pour renouveler l'approche romanesque du national-socialisme. Deux de ses romans sont disponibles en français, Voix de la nuit (Flughunde, traduit de l'allemand par François Mathieu, Calmann-Lévy, 1995) et Kaltenburg (Cologne, Dumont, 2008, traduit par Cécile Wajsbrot, Métailié, 2010). Afin de donner une idée de l'impact de Simon sur la jeune génération des romanciers allemands, nous avons choisi pour le volume Lectures allemandes de Claude Simon (Septentrion, 2013) deux textes de Beyer: un essai sur les pouvoirs du langage intitulé "Salive" (1998), ayant pour point de départ un souvenir que Simon raconte dans Le Jardin des Plantes, ainsi que le dernier chapitre du roman Spione (Cologne, DuMont, 2000; "Espions "), dans lequel la " confabulation " à partir de quelques documents photographiques fait penser à L'Acacia et au neuvième chapitre d'Histoire de Simon. L'essai que Laurent Cassagnau a maintenant traduit pour le présent numéro des Cahiers Claude Simon a été la contribution de Beyer au colloque "Claude Simon en Allemagne " qui a eu lieu le 30 novembre 2013 à la Bibliotheca Reiner Speck à Cologne. L'écrivain y revient sur les visites faites avec sa compagne, la photographe Jacqueline Merz, à Mühlberg sur Elbe au Stalag IV B vers la fin des années quatrevingt. Mais au lieu de porter sa réflexion sur les traces matérielles ou imaginaires de ce que vécut Simon dans ce lieu, Beyer propose une lecture surprenante qui situe les romans de Simon dans l'histoire du vingtième siècle à partir des dates de publication de ses romans. Ainsi, Le Palace (1962) serait à lire dans le contexte de la guerre d'Algérie et L'Acacia (1989) dans celui de la fin de la guerre froide. C'est à Mühlberg et après son retour en France que Simon a, selon Beyer, commencé à superposer les images de la mémoire et du présent de l'écriture, d'abord en dessinant, puis en écrivant. Dans son essai, Beyer ajoute à ceci une autre couche d'images où s'entremêlent ses lectures des romans de Simon et son imaginaire allemand de la guerre, des camps de concentration et de la guerre froide. De cette manière, il montre comment les romans de Simon sont vraiment devenus pour lui " un outil de perception du monde par le biais de l'écriture". 